\title{
Several new Japanese guidelines issued
}

\author{
Michiaki Yamakage $^{1}$ (ID
}

Received: 13 February 2017 / Accepted: 15 February 2017 / Published online: 24 February 2017

(C) Japanese Society of Anesthesiologists 2017

Keywords Smoking cessation · Medication error . Malignant hyperthermia $\cdot$ Guideline

Numerous evidence-based Japanese guidelines have recently been issued by different committees. Although each has been written in Japanese for members of the Japanese Society of Anesthesiologists (JSA), we are publishing English translations in this journal with the purpose of disseminating the information worldwide. In this issue, we publish three sets of guidelines. All are extremely interesting, and we are confident that they will provide useful information not only for members of the JSA, but also for anesthesiologists worldwide.

The first is the guideline for perioperative smoking cessation [1]. Its publication is undeniably a little behind the times. A declaration on quitting smoking was published by the JSA in 2008, but was not widely disseminated in clinical practice. Japan has a comparatively high rate of smoking for a developed country, and stopping smoking during the perioperative period is considered important; if possible, the opportunity should be taken to give up altogether. This guideline has three points-(1) quitting smoking before surgery may decrease perioperative complications, (2) quitting smoking at any point is always beneficial, and (3) the longer an individual does not smoke, the better the possibility of reducing perioperative complications. We leave the detailed description of the benefits of

Michiaki Yamakage

yamakage@sapmed.ac.jp

1 Department of Anesthesiology, Sapporo Medical University School of Medicine, South 1, West 16, Chuo-ku, Sapporo, Hokkaido 060-8543, Japan quitting smoking to the guideline itself; however, anesthesiologists responsible for managing the perioperative safety of patients should be aware of these and make efforts to encourage patients to stop smoking before surgery. I must confess that I myself smoked when I was young. People who have previously been smokers but have now quit are troubled by the smell of cigarette smoke around them. This guideline also refers to the effects of passive smoking. I have recently come to enjoy smoking cigars when drinking alcohol. Cigars are something to be savored in the mouth, but do they also affect perioperative complications?

The next guideline to be introduced concerns the prevention of medication errors [2]. Unlike internists, we anesthesiologists handle only a very limited range of drug classes, but most are poisons or powerful pharmacotherapeutic agents, and incorrect administration may result in serious complications. Human error is frequently involved, and simply saying 'Just be more careful next time' is probably not a fit response in terms of contemporary risk management. This guideline emphasizes recommendations for drug syringe labels in particular. Detailed stipulations for these labels are provided, specifying that they should (1) be an appropriate size, (2) be resistant to peeling off, (3) be resistant to fading, (4) provide the appropriate content, and (5) be printed in black lettering in an easily legible font. Interestingly, the guideline also stipulates the background color, with a clear explanation of the drugs and antagonists used. I am also in favor of this, but if these matters are decided at the level of individual institutions, then the possibility of mistaken administration will remain, similar to the difficulties that would be faced if the color of traffic lights were to vary between prefectures or countries. However, if a mistake has already been made at the drug preparation stage, the color of the label may be meaningless. To prevent this from happening, use of prefilled or colored 
syringes may be preferable. I intend to take this opportunity to review the syringes used in the operating room at my own hospital.

The final guideline is the JSA Guideline for the management of malignant hyperthermia ( $\mathrm{MH})$ crisis [3]. As the epidemiology of this condition varies between countries and regions, the guidelines should reflect actual situations in individual nations. The distinctive feature of this guideline is that the treatments for $\mathrm{MH}$ and postoperative $\mathrm{MH}$ (PMH) are clearly set out as bullet points, facilitating use in an actual crisis when the situation on the ground may be very confused. The way in which the answers to possible queries are summarized in a Q\&A format will also be useful in improving understanding. This guideline not only deals with the treatment of an MH crisis, but also includes a review-type element that provides an understanding of the underlying physiopathology. The enthusiasm of the working group involved is palpable.

Of course, we hope that not only will these guidelines be of use to JSA members when they have the opportunity to look at them in the course of patient safety management in actual clinical practice, but that their publication in their entirety in English and free of charge will mean that they will be seen by, and of use to, many people around the world. The European Society of Anesthesiologists (ESA) and the American Society of Anesthesiologists (ASA) have also published numerous guidelines, and the extent of variations between different countries and regions is interesting. This is because even though medicine is supposed to be a science, as a discipline it is concerned with human beings, and of course guidelines will vary as a result of differences in race, culture, types of drugs and devices available for use, and social situations. Comparing these guidelines and going back to the underlying research will be beneficial in terms of accumulating further evidence.

\section{References}

1. Safety Committee of Japanese Society of Anesthesiologists. A guideline for perioperative smoking cessation. J Anesth. 2017 (in press).

2. Safety Committee of Japanese Society of Anesthesiologists. Preventing medication errors in the perioperative setting: recommendations on drug syringe labels. J Anesth. 2017 (in press).

3. Malignant Hyperthermia Working Group and Safety Committee of Japanese Society of Anesthesiologists. JSA guideline for the management of malignant hyperthermia crisis 2016. J Anesth. 2017 (in press). 